Article

\title{
Novel spa and Multi-Locus Sequence Types (MLST) of Staphylococcus Aureus Samples Isolated from Clinical Specimens in Korean
}

\author{
Yae Sung Mun ${ }^{1}$ and You Jin Hwang ${ }^{1,2,3, *}$ \\ 1 Department of Life science, College of Bio-nano technology, Gachon University, 191, Hambangmoe-ro, \\ Yeonsu-gu, Incheon 21936, Korea; yae01@naver.com \\ 2 Department of Health Sciences and Technology, GAIHST, Gachon University, Incheon 21999, Korea \\ 3 Department of Biomedical Engineering, College of Health Science, Gachon University, 191, Hambangmoe-ro, \\ Yeonsu-gu, Incheon 21936, Korea \\ * Correspondence: gene@gachon.ac.kr; Tel.: +82-032-820-4545; Fax: +82-032-820-4449
}

Received: 30 September 2019; Accepted: 22 October 2019; Published: 29 October 2019

\begin{abstract}
Staphylococcus aureus bacteremia is one of the most frequent and severe bacterial infections worldwide. The increased incidence of $S$. aureus infections with a diverse pattern of $S$. aureus protein A (spa) types across different geographic regions is a global challenge. This study investigated a novel spa type of methicillin-resistant $S$. aureus in a clinically isolated specimen. A total of 109 clinical $S$. aureus samples were subjected to 19 sets of antimicrobial susceptibility testing using the Kirby-Bauer disk diffusion method. Molecular typing was performed with S. aureus protein A (spa) and multi-locus sequence types (MLST) via polymerase chain reaction and sequencing. The methicillin-resistant $S$. aureus samples in our study accounted for 55.05\% (60/109) of the total. A novel spa type was detected in five (5/60) strains. This gh22 isolate was identified in antimicrobial susceptibility tests of 15 kinds of antibiotics. Antibiotic resistance genes included mecA, TEM, aac(6')-aph(2"), ermA, and tetM. Eleven $S$. aureus samples were classified as $t 2460, t 338, t 324, t 693$, five unknown spa types (new spa types), and undefined MLST (novel MLST). We report a high prevalence rate of $t 2460$ methicillin-resistant S. aureus samples in our country. Additionally, novel spa gh22, MLST ST4613, and clonal compact CC5-type strains (T1:M1:B1:B1:M1:E1:K1, r26:r17:r34:r34:r17:r13:r16, mlst;1:4:1:4:559:495:10) showing multidrug resistance were identified among $S$. aureus samples.
\end{abstract}

Keywords: spa typing; spa; MLST; multidrug-resistance; methicillin-resistant Staphylococcus aureus (MRSA)

\section{Introduction}

Methicillin-resistant Staphylococcus aureus (MRSA) is a prominent pathogen that causes severe infections in both healthcare and community settings [1]. MRSA is associated with considerable infection worldwide [2]. Resistance to antimicrobial agents, especially nosocomial pathogens, has become one of the most serious challenges worldwide. Excessive therapeutic application of antimicrobial agents in both humans and animals has contributed to the development of widespread antibiotic resistance in bacteria [3,4]. Multidrug-resistant $S$. aureus is a serious public health concern warranting medical attention [5].

Epidemiological studies are an essential component in the study of clonality, evolutionary pathways, genetic diversity of pathogens, and the spread of S. aureus infections [6]. Various molecular typing methods can be used to type MRSA isolates [7]. Spa typing is based on the number of tandem repeats and the sequence variation in region $X$ of the protein $A$ gene. The spa gene contains three distinct regions: $\mathrm{Fc}, \mathrm{X}$, and $\mathrm{C}[8,9]$. Based on a literature review, the spa type distribution of MRSA strains isolated 
from patients in different geographic locations worldwide exhibits diverse patterns [10]. Pulsed-field gel electrophoresis (PFGE) with high discriminatory power is the documented gold standard among the various DNA sequencing methods available [11,12]. However, due to its labor-intensive methodology, difficulties associated with data exchange between laboratories, and the need for inter-laboratory standardization, PFGE has been replaced by multi-locus sequence typing (MLST) and staphylococcal protein A (spa) typing [8]. Of these, spa typing, which relies only on the assessment of the number and sequence variation in repeats at the $X$ region of the spa gene, exhibits excellent discriminatory power and has become a user-friendly and cost-effective typing tool with a standardized nomenclature [5,12-14].

MLST is an effective tool for evolutionary investigation and differentiation of isolates according to nucleotide variations in seven housekeeping genes [15]. MLST has proven very useful for macroepidemiology and evolutionary studies [16]. However, spa typing is also both easier and less costly to perform than MLST or PFGE. Therefore, spa typing combined with unambiguous typing results of MLST can reduce costs for laboratories over time.

Country-specific data relating to the distribution of diverse spa and MLST types have yet to be clearly reported. This study elucidated the patterns of antibiotic resistance based on antibiotic sensitivity testing and the molecular epidemiology and characterization, including new spa and novel MLST typing approaches in S. aureus samples collected from clinical sources at Gil Hospital, Incheon, Korea.

\section{Results}

We tested for antimicrobial susceptibility using Kirby-Bauer disc diffusion and established the isolates as resistant or susceptible to antimicrobial agents based on the diameters of the inhibition zone. Our susceptibility testing showed that 56.88\% (60/109) of S. aureus samples were resistant to methicillin. We selected 13 specific samples and compared the susceptibility results via disc diffusion. The antibiotic-resistant genes in S. aureus samples were identified using the PCR assay (Table 1).

Table 1. Phenotypic antibiotic resistance patterns and rates of antibiotic-resistant genes in S. aureus samples.

\begin{tabular}{|c|c|c|c|c|c|c|c|c|c|c|c|c|c|c|c|c|c|c|c|}
\hline Samples & Meth & Pen & Kan & Erh & Gen & Tet & Strep & Van & Chlo & AML & TC & PRL & FEP & CTX & CAZ & IMI & ETP & MRP & ATM \\
\hline gh2 & 0 & 0 & 0 & 0 & 0 & 10 & 8 & 15 & 22 & 20 & 14 & 0 & 0 & 0 & 0 & 7 & 0 & 10 & 0 \\
\hline gh22 & 0 & 0 & 0 & 0 & 0 & 11 & 8 & 14 & 21 & 14 & 7 & 0 & 0 & 0 & 0 & 0 & 0 & 7 & 0 \\
\hline gh11 & 16 & 0 & 0 & 20 & 0 & 23 & 8 & 14 & 25 & 25 & 25 & 25 & 25 & 25 & 25 & 25 & 25 & 25 & 0 \\
\hline gh19 & 0 & 0 & 0 & 0 & 0 & 12 & 10 & 14 & 25 & 12 & 0 & 0 & 0 & 0 & 0 & 0 & 0 & 0 & 0 \\
\hline gh20 & 15 & 0 & 12 & 20 & 12 & 20 & 8 & 12 & 23 & 24 & 25 & 25 & 25 & 25 & 25 & 25 & 25 & 25 & 0 \\
\hline gh21 & 0 & 8 & 12 & 0 & 10 & 8 & 8 & 13 & 20 & 18 & 13 & 0 & 0 & 0 & 0 & 0 & 0 & 9 & 0 \\
\hline gh34 & 18 & 9 & 17 & 20 & 15 & 23 & 10 & 13 & 22 & 25 & 25 & 25 & 25 & 25 & 25 & 25 & 25 & 25 & 0 \\
\hline gh36 & 0 & 0 & 17 & 23 & 15 & 25 & 10 & 15 & 25 & 15 & 18 & 12 & 22 & 18 & 15 & 14 & 18 & 20 & 0 \\
\hline gh5-t2460 & 0 & 0 & 11 & 0 & 11 & 10 & 0 & 15 & 22 & 14 & 10 & 10 & 0 & 0 & 0 & 0 & 0 & 8 & 0 \\
\hline gh7-t338 & 16 & 0 & 14 & 9 & 12 & 25 & 8 & 14 & 24 & 25 & 25 & 25 & 25 & 25 & 25 & 25 & 25 & 25 & 25 \\
\hline gh27-t2460 & 0 & 0 & 0 & 22 & 0 & 25 & 9 & 14 & 23 & 14 & 20 & 14 & 20 & 20 & 14 & 25 & 25 & 25 & 0 \\
\hline gh97-t324 & 0 & 10 & 8 & 27 & 18 & 28 & 15 & 19 & 29 & 13 & 8 & 9 & 15 & 14 & 0 & 0 & 0 & 14 & 0 \\
\hline gh100-t693 & 19 & 20 & 21 & 29 & 20 & 28 & 14 & 16 & 29 & 18 & 20 & 14 & 15 & 15 & 9 & 25 & 20 & 25 & 0 \\
\hline
\end{tabular}

We tested the following 19 antibiotic discs (Liofilchem, Roseto degli Aburzzi, Italy). We measured the diameter $(\mathrm{mm})$ of the inhibition zones and determined each isolate as resistant or susceptible to antimicrobial agents based on CLSI guidelines and criteria and Liofilchem quality control.

Our susceptibility testing also showed (Table 1) that two S. aureus samples (gh11, gh20) were susceptible to penicillin G, tetracycline, erythromycin, and extended spectrum antibiotics, including cephalosporins, cabapenems, and monobactams. Four samples $(g h 2, g h 22, g h 19, g h 21)$ exhibited resistance against methicillin, penicillin $\mathrm{G}$, erythromycin, and partially extended spectrum cephalosporins, cabapenems, and monobactams (Table 1).

Three samples (gh2, gh22, and gh19) tested PCR-positive for TEM, mecA, SCCmec type II, $a a c\left(6^{\prime}\right)$-aph (2"), tet $\mathrm{M}$, and ermA genes (Table 2). Sample gh21 was tested positive for bla ${ }_{\mathrm{TEM}}, \operatorname{mec} A$, SCCmec type II, tet $\mathrm{M}$, and erm A, but not for aac (6')-aph(2"), kanamycin, or gentamicin antibiotic genes. 
Table 2. Comparison of previously known Ridom types and novel spa types in S. aureus samples.

\begin{tabular}{|c|c|c|c|c|c|}
\hline $\begin{array}{c}\text { samples/ } \\
\text { Ridom type }\end{array}$ & $\begin{array}{c}\text { repeat } \\
\text { sequence }\end{array}$ & $\begin{array}{c}\text { repeat } \\
\text { units }\end{array}$ & length & spa repeat sequence & spa repeat sequence \\
\hline gh2 & 41 & 7 & 168 & T1:M1:B1:B1:M1:E1:* & r26:r17:r34:r34:r17:r13:r314 \\
\hline gh22 & 31 & 7 & 168 & T1:M1:B1:B1:M1:E1:K1 & r26:r17:r34:r34:r17:r13:r16 \\
\hline gh11 & 41 & 8 & 192 & U1:G2:M1:F1:*:B1:L1:B1 & r07:r06:r17:r21:r81:r34:r22:r34 \\
\hline gh19 & 41 & 7 & 168 & T1:M1:B1:B1:M1:E1:* & r26:r17:r34:r34:r17:r13:r314 \\
\hline gh20 & 44 & 6 & 144 & U1:J1:G1:F1:*:* & r07:r23:r12:r21:r298:r254 \\
\hline gh21 & 47 & 10 & 240 & T1:M1:B1:B1: :*:*:**:*:* & r26:r17:r34:r34:r298:r377:r298:r298:r298:r314 \\
\hline gh5-t2460 & 25 & 10 & 240 & T1:M1:B1:B1:M1:D1:M1:M1:M1:K1 & r26:r17:r34:r34:r17:r20:r17:r17:r17:r16 \\
\hline gh7-t338 & 42 & 7 & 168 & W1:F1:K1:A1:O1:M1:Q1 & r15:r21:r16:r02:r25:r17:r24 \\
\hline gh27-t2460 & 35 & 10 & 240 & T1:M1:B1:B1:M1:D1:M1:M1:M1:K1 & r26:r17:r34:r34:r17:r20:r17:r17:r17:r16 \\
\hline gh97-t324 & 45 & 10 & 240 & U1:J1:G1:G1:M1:D1:M1:G1:G1:M1 & r07:r23:r12:r12:r17:r20:r17:r12:r12:r17 \\
\hline gh100-t693 & 48 & 1 & 24 & $\mathrm{U} 1$ & r07 \\
\hline
\end{tabular}

* Ridom is spaserver.ridom.de, CLUSTAL 2.1 is multiple sequence alignment, gh is Gil Hospital, ND is not determined.

Sequence analysis showed 13 specific samples and revealed different spa types among the 11 clinical samples of $S$. aureus, including four known spa types (t2460(2), t338, t324, and $t 693)$ and six new spa types ( $g h 2, g h 11, g h 19, g h 20, g h 21$, and $g h 22)$, as defined by the Ridom SpaServer. These results, involving multiple patterns among the distribution of spa types, are presented in Figure 1 and Table 2.
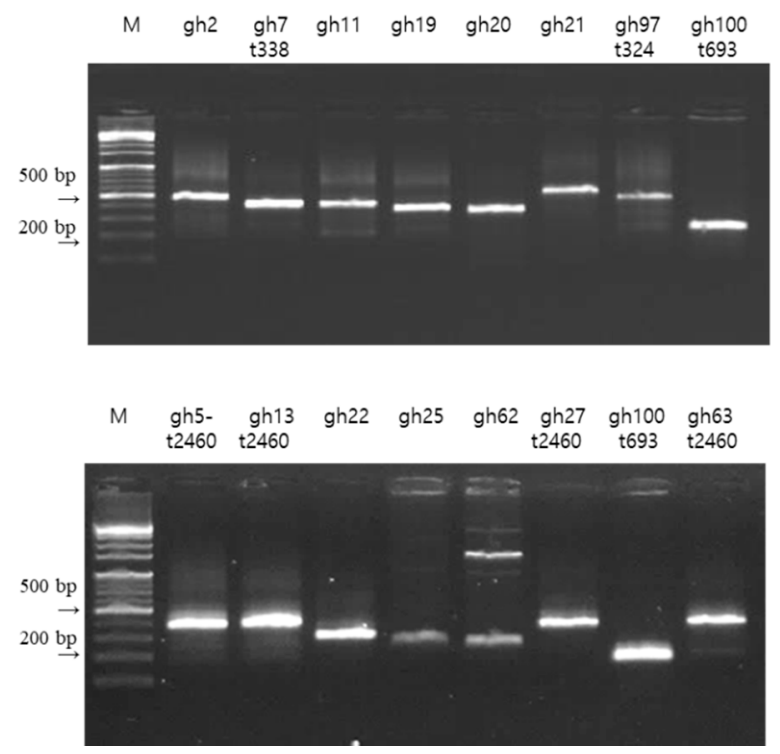

Figure 1. PCR amplification of the spa type gene. The PCR results were visualized by agarose gel electrophoresis. Lane M, 100 bp DNA ladder. Gh2 to gh100 represent PCR-amplified spa strains (180-600 $\mathrm{bp})$.

Six new spa types were identified in Ridom SpaServer. Spa type $t 2460$ was the predominant spa type in our samples (data not shown here), and spa sequence analysis showed five different genotypes among 11 specific MRSA isolates (Figure 2). Four spa types, namely t2460, t338, t324, and t693, were distinguished using the Ridom method in this study (Table 2).

We compared a total of 35 known spa strains of MRSA, with 13 of our isolate samples obtained from patient samples in Korea. A phylogeny review of 46 total split circles depicting the phylogeny tree revealed a region containing 35 known isolates and 11 samples (Figure 3) (multiple sequence alignment by CLUSTALW, http://rest.genome.jp/link/. Kyoto University Bioinformatics Center, Kyoto, Japan). 


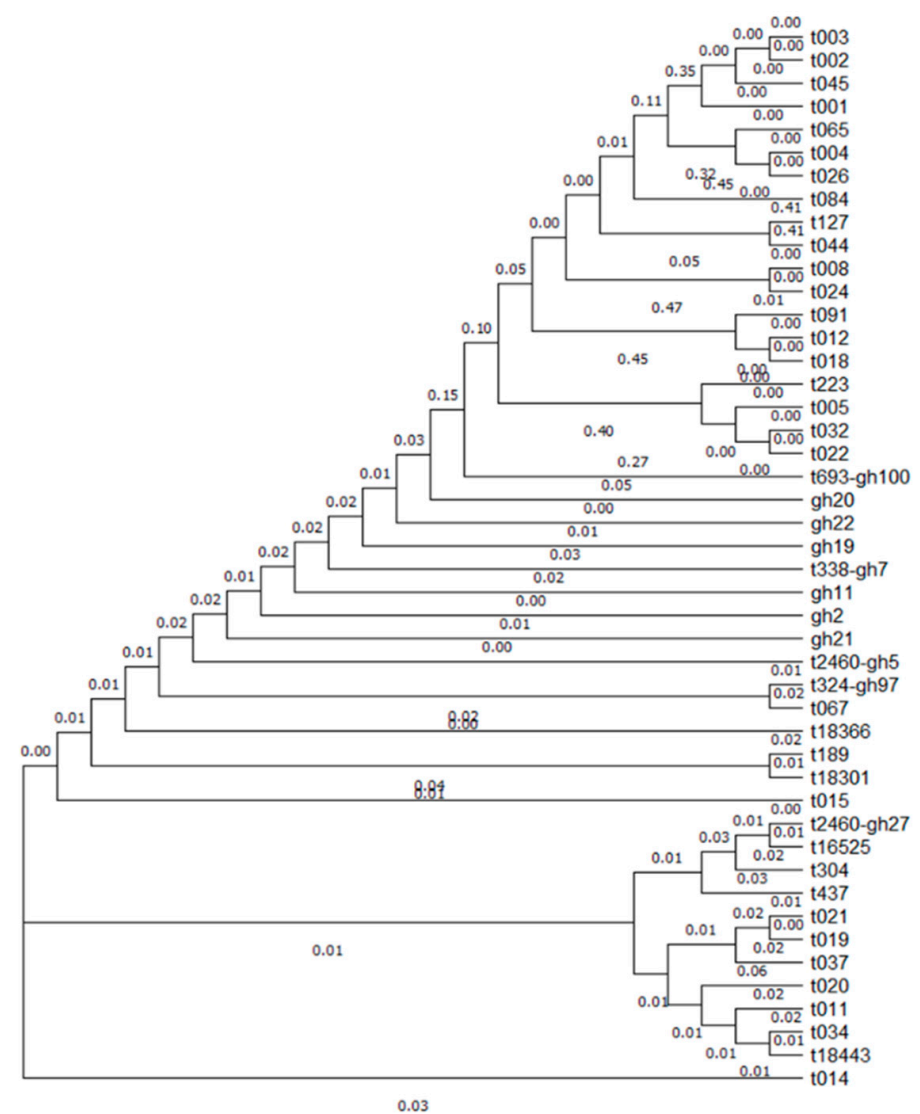

Figure 2. A split circle showing the similarity of the DNA sequence cluster. A total of 35 current strains of methicillin-resistant Staphylococcus aureus (MRSA) and 11 isolated strains from patient samples in Korea. The image provides an overview of the 46 total split circles and a detailed view of the region containing the 26 highly similar samples, which includes 15 existing and 11 new isolates (Multiple Sequence Alignment by CLUSTAL2.1, http://rest.genome.jp/link/. Kyoto University Bioinformatics Center, Kyoto, Japan).

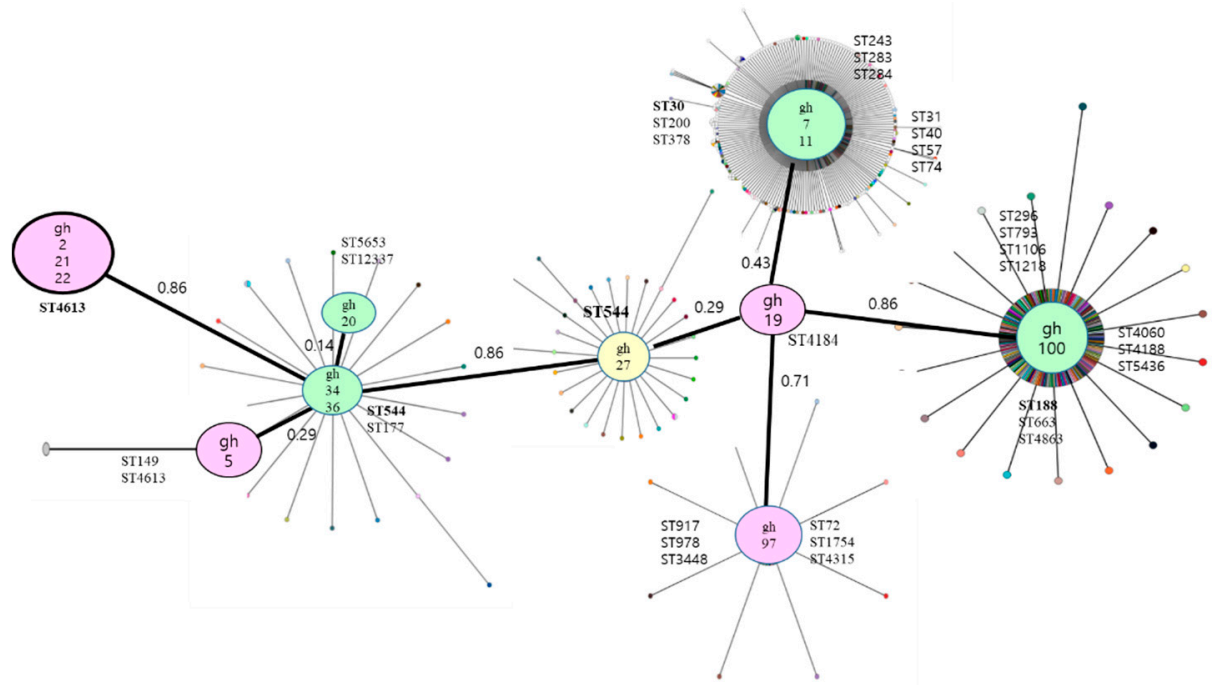

Figure 3. Output of an eBURST analysis of S. aureus sequence types (ST) in the multi-locus sequence typing (MLST) database, listed as being MRSA of human origin. The group definition was set to "zero alleles in common" in order to allow visualization of all STs on a single diagram. The diameters of the solid spots are proportional to the occurrences of the STs in the MLST database (i.e., a large spot means that a large number of isolates with that ST have been entered into the database). Red is multi-drug resistance (MDR), green is no MDR. 
Spa gene samples $g h 2$ and $g h 19$ showed similar sequences and a single partially different sequence carrying the same repeat sequence of Ridom types. Sample $g h 22$ was similar to $g h 2$ and $g h 19$, except for a single variation in a repeat sequence. The repeat numbers assigned by the SpaServer were 26-17-34-34-17-13-16 and 26-17-34-34-17-13-314 (Table 2). Further, gh2, gh19, and gh22 were the only six nucleotide sequence variations carrying a single repeat sequence variation and similar superantigen profiles, suggesting that the strains $g h 2$, gh19, and $g h 22$ were closely linked. Additionally, gh11, gh20, and $g h 21$ represented different repeat sequences. The repeat numbers assigned by the SpaServer were 07-06-17-21-81-34-22-34, 07-23-12-21-298-254, and 26-17-34-34-298-377-298-298-298-314.

MLST analysis showed 13 specific samples. They were differently revealed and undefined MLST types (Table 3). Three strains ( $g h 2, g h 21, g h 22)$ exhibited ST4613, as well as clonal complex CC5. Ten strains did not have defined MLST and had different clonal complexes.

Table 3. Comparison of novel strains in S. aureus samples revealed through MLST.

\begin{tabular}{|c|c|c|c|c|c|c|c|c|c|}
\hline Samples & $\operatorname{arcC}$ & aroE & $g l p F$ & $g m k$ & $p t a$ & tpi & yqiL & $M L S T$ & $\begin{array}{c}\text { Clonal } \\
\text { complex }\end{array}$ \\
\hline$g h 2$ & 1 & 4 & 1 & 4 & 559 & 495 & 10 & ST4613 & CC5 \\
\hline gh22 & 1 & 4 & 1 & 4 & 559 & 495 & 10 & ST4613 & CC5 \\
\hline gh11 & 1 & 4 & 1 & $N D$ & $N D$ & $N D$ & $N D$ & ND & [CC1] \\
\hline gh19 & 1 & 4 & 1 & 4 & 559 & 134 & 10 & $N D$ & [CC5] \\
\hline gh20 & 3 & 1 & 1 & 8 & 322 & 495 & 295 & $N D$ & [CC1] \\
\hline gh21 & 1 & 4 & 1 & 4 & 559 & 495 & 10 & ST4613 & CC5 \\
\hline gh34 & 1 & 4 & 1 & 8 & 4 & 497 & 76 & $N D$ & $N D$ \\
\hline gh36 & 177 & 4 & 1 & 8 & 4 & 368 & 76 & $N D$ & $N D$ \\
\hline$g h 5-t 2460$ & 1 & 4 & 1 & 4 & 559 & 41 & 10 & $N D$ & [CC45] \\
\hline$g h 7-t 338$ & 2 & 2 & 95 & 185 & 6 & 201 & 500 & $N D$ & [CC1] \\
\hline$g h 27-t 2460$ & 1 & 4 & 1 & 185 & 4 & 497 & 76 & $N D$ & [CC1] \\
\hline$g h 97-t 324$ & 1 & 4 & 1 & 8 & 4 & 497 & 3 & $N D$ & [CC 8$]$ \\
\hline gh100-t693 & 3 & 1 & 1 & 8 & 1 & 134 & 295 & $N D$ & [CC1] \\
\hline
\end{tabular}

The phylogenetic MLST tree showed minimum spanning based on MLST allelic profiles. Nine category samples were neighbor-joining, and each sample was compared with subtype sequences (Figure 3). The $g h 7$ and $g h 11$ samples were arcC, aroE and glpF different sequences but had the same type of pubMLST classification. The $g h 19$ strain showed different similarity distances to $g h 7, g h 11$, $g h 27, g h 97$, and $g h 100$ strains, but was more similar than $g h 2, g h 5$, and $g h 20$ strains (Figure 3). Figure 3 shows the MLST sequence variant distance and antibiotic resistance. The red mark indicates multiple antibiotics resistance and green indicates no or little resistance.

\section{Discussion}

The widespread emergence of MRSA (S. aureus) is a great public health challenge. Currently, the spread of MRSA limits therapeutic options and causes severe morbidity and mortality in hospitalized patients [2,3]. The prevalence of MRSA also varies widely in different geographic regions worldwide $[3,17,18]$. Molecular spa typing of $S$. aureus isolates is important for the identification of dominant strains associated with disease outbreaks and drug resistance, and also in tracing the transmission chain and source of infection [4,19]. In this study, 11 specific MRSA isolates were found that had not been reported by other studies from Korea. According to the results of drug susceptibility testing, the expected MRSA strains were characterized by higher rates of resistance to antimicrobial agents, including penicillin G, tetracycline, erythromycin, extended spectrum antibiotics, cephalosporins, cabapenems, and monobactams. The gh2, gh22 and gh19 MRSA strains showed high rates of antibiotic resistance and tested positive for antibiotic-resistant genes.

The SpaServer identified 18,514 different spa types as of January 28, 2019. Among the 109 MRSA and MSSA strain isolates, we found five novel spa types. The spa type $t 2460$ was the most dominant type $(20 / 60,33.3 \%)$ in the studied population. Interestingly, the 2460 genotype was only found among 
the MRSA isolates. The isolated strain $t 2460$ exhibiting SCCmec type II was predominant among the investigated MRSA isolates. Similar studies reported individual t2460 MRSA isolates in human hosts from China and Korea [20-22].

In this study, we identified new spa types $g h 2, g h 11, g h 19, g h 20, g h 21, g h 22$, and SCCmec type II in our samples. Four spa types, namely $t 2460, t 338, t 324$, and $t 693$, were distinguished using the Ridom type method in this study (Table 3). In another international study, the prevalence of $t 037$, which was the most frequent spa type in 2000-2001, was reduced in 2007-2008. By contrast, $t 2460$ was the most frequent spa type in 2007-2008, as shown in [21]. The increased prevalence of $t 2460$ strain in 2007-2008 was not related to the outbreak. Similarly, t002, t062, t601, and t2460 exhibit spa-CC002 [21,22]. In Asian countries, the most predominant clones in the region included ST59-MRSA-SCCmec type IV-spa type $t 437$ in Taiwan, Hong Kong, Vietnam, and Sri Lanka; ST30-MRSA-SCCmec type IV-spa type $t 019$ in the Philippines; and ST72-MRSA-SCCmec type IV-spa type $t 324$ in Korea [23]. Some of the health care-associated (HA-MRSA) isolates from Taiwan, the Philippines, and Korea showed the same genotypic characteristics as community-associated MRSA (CA-MRSA) isolates in these countries [6,24,25]. In China, t030 was the predominant spa type [26]. However, it was also reported in Iran as the fifth most common spa type. Moreover, 037 was the second most common spa type in Asia, and was reported in several Asian countries (Korea, China, Taiwan, Iran, and Malaysia) [21-28].

Previous MLST results of molecular typing showed only a few sequence types (ST) in Korea. In these results, our samples were the similar types ST4613, ST544, ST188, and ST30. Cha et al. reported on ST239 and the presence of only a few major MRSA strains in the clonal spread of MRSA in Korea [8]. Testing for the prevalence of MLST types showed that ST5-SCCmec II- 2460 and ST5-SCmec II- $t 002$ $(68.4 \%)$ were the predominant clones, followed by ST72-SCCmec IV-t324 (15.8\%), ST239-SCCmec III- $t 037$ $(10.5 \%)$, and ST1-SCCmec IV-t286 (3.9\%) [29].

Both ST59 and ST338 belong to CC59, which have shown the prevalent CA-MRSA clone in China and other Asian countries [30,31]. Along with three CC59 MRSA isolates, we also identified a single ST59 MSSA isolate among the Ready-to-eat (RTE) food samples. In China, ST59 and ST338 were the first and second most dominant sequence types (STs) in cases of pediatric community-acquired pneumonia [32]. In recent years, community-associated MRSA (CA-MRSA) has emerged as an important cause of infection, with geographical differences among strains-ST1 (USA400) and ST8 (USA300) exist in North America, ST80 is found in Europe, ST59 is found in the Asia-Pacific region, and ST30 is noted worldwide [33,34]. Among the MSSA strains, ST188, ST72, ST5, and ST30 occurred most frequently, consistent with previous findings in Korea $[17,35,36]$. ST72, which is a major CA-MRSA clone in South Korea, was distinct from those that have spread throughout Asia (ST30, ST59, and ST338) or internationally [36]. The Korean CA-MRSA strain has emerged in the community and has recently been spreading in healthcare settings [36,37].

In this study, five new spa and ten new MLST types were reported for the first time and did not represent the currently known strains of MRSA, MSSA, or antibiotic susceptibility. In summary, the results showed that 2460 was one of the major spa types along with SCCmec type II in Korea. Our results demonstrated differences in the regional spa and MLST types of Korea compared with those originating in Asia, Europe, and other geographic locations.

\section{Materials and Methods}

\subsection{Materials and Bacterial Isolates}

Our study was conducted at Gachon University Gil Medical Center in Incheon, South Korea, between April 2016 and June 2018. The research was approved by the ethics committee of Gil Hospital, Gachon University of Medicine. Sample identification and antimicrobial susceptibility testing of $S$. aureus isolated from blood agar plates (Shin Yang Chemical Co., Ltd.'s media., Seoul, Korea) were performed using a MicroScan Pos Breakpoint Combo panel type 28 (PBC28; Beckman Coulter, West Sacramento, CA, USA). Sample strains were streaked onto sheep blood agar (Sinyang Diagnostics, 
Seoul, Korea) and transported to our laboratory after culture. One colony was picked from each blood agar plate and incubated in lysogeny broth with shaking $(80 \mathrm{rpm})$ at $37^{\circ} \mathrm{C}$ overnight. Isolates were preserved in $20 \%$ glycerol $(\mathrm{vol} / \mathrm{vol})$ and stored in a $-80^{\circ} \mathrm{C}$ freezer until further use.

\subsection{Antimicrobial Susceptibility Testing}

We tested for antimicrobial susceptibility using the Kirby-Bauer disc diffusion method described by Clinical and Laboratory Standard Institute (CLSI) guidelines, 2013 [38]. Each bacterial suspension was adjusted to McFarland 0.5 turbidity, swabbed onto Muller-Hinton agar, and incubated in the presence of antibiotic discs at $35{ }^{\circ} \mathrm{C}$ for $18 \mathrm{~h}$.

We tested the following 19 antibiotic discs from Liofilchem (Liofilchem, Roseto degli Aburzzi, Italy): penicillin G (10 IU), methicillin (5 $\mathrm{g})$, kanamycin $(30 \mu \mathrm{g})$, gentamicin $(10 \mu \mathrm{g})$, streptomycin $(10 \mu \mathrm{g})$, tetracycline $(30 \mu \mathrm{g})$, erythromycin $(15 \mu \mathrm{g})$, vancomycin $(30 \mu \mathrm{g})$, chloramphenicol $(30 \mu \mathrm{g})$, amoxicillin $(25 \mu \mathrm{g})$, ticarcillin $(75 \mu \mathrm{g})$, piperacillin $(100 \mu \mathrm{g})$, cefepime $(30 \mu \mathrm{g})$, cefotaxime $(30 \mu \mathrm{g})$, ceftazidime $(30 \mu \mathrm{g})$, imipenem $(10 \mu \mathrm{g})$, ertapenem $(10 \mu \mathrm{g})$, meropenem $(10 \mu \mathrm{g})$, and aztreonam (30 $\mu \mathrm{g})$. We measured the diameters of the inhibition zones and determined each isolate as resistant or susceptible to antimicrobial agents based on CLSI guidelines and Liofilchem quality control parameters. We obtained S. aureus control strain Staphylococcus aureus ATCC 29,213 (Korean Culture Center of Microorganisms, Seodaemun-gu, Seoul, Korea).

\subsection{Genomic DNA Extraction}

The QIAamp DNA Mini Kit (Cat No: 51306, Qiagen GmbH, Hilden, Germany) was used for genomic DNA extraction according to the manufacturer's instructions. DNA concentrations were determined using a NanoDrop ${ }^{\mathrm{TM}}$ spectrophotometer (Thermo Fisher Scientific, Waltham, MA, USA).

\subsection{Identification of MecA, Bla $a_{T E M}$, and the Detection of Genes Associated with Antimicrobial Resistance by Multiplex PCR}

The PCR primers used to detect $m e c A, b l a_{\mathrm{TEM}}$, and antimicrobial resistance genes are listed in references [39-41]. The following reaction mixture was added to each sample: 10 pmol of each primer, 2 $\mu \mathrm{L}$ DNA $(100 \mathrm{ng})$, and $10 \mu \mathrm{L} \mathrm{iQ}^{\mathrm{TM}}$ cyber $(\mathrm{SYBR}){ }^{\circledR}$ Green supermix $(2 \times$ reaction buffer with $\mathrm{dNTP}(\mathrm{dATP}$, dCTP,dTTP, dGTP)s, iTaq DNA polymerase, SYBR ${ }^{\circledR}$ Green I, fluorescein, and stabilizers; Bio-Rad, Hercules, CA, USA). The volume was adjusted to $20 \mu \mathrm{L}$ with autoclaved, triple-distilled water. The PCR conditions in the thermal cycler (TC-512, UK) were as follows: $94{ }^{\circ} \mathrm{C}$ for 3 min, followed by 35 cycles of denaturation at $94{ }^{\circ} \mathrm{C}$ for $30 \mathrm{~s}$, annealing at $56{ }^{\circ} \mathrm{C}$ for $30 \mathrm{~s}$, and extension at $72{ }^{\circ} \mathrm{C}$ for $45 \mathrm{~s}$. The reaction was ended with a final extension step at $72{ }^{\circ} \mathrm{C}$ for $10 \mathrm{~min}$. PCR products were subjected to electrophoresis using 2\% agarose gel in $1 \times$ TBE buffer at $100 \mathrm{~V}$ for $25 \mathrm{~min}$. The $100 \mathrm{bp}$ DNA ladder (Bioneer, Daejeon, Korea) was used as a molecular size marker. PCR products in gels were visualized with a Safe Green loading dye (Applied Biological Materials Inc., Vancouver, BC, Canada).

\section{5. spa, MLST Typing, and Phylogenetic Analysis}

The spa typing was performed as described by Harmsen et al. (8). The polymorphic $\mathrm{X}$ region of the spa gene was amplified using primers spa1095F (5'-AGACGATCCTTCGGTGAGC-3') and spa1517R (5'-GCTTTTGCAATGTCATTTACTG-3'). PCR spa gene products were subjected to DNA sequencing of both strands by Macrogen (Seoul, Korea). The sequences were analyzed using Ridom StaphType v2.0.3 software (Ridom $\mathrm{GmbH}$ ). The guidelines derived from the Ridom SpaServer database (http://www.spaserver.ridom.de) were used to assign the edited sequences to the particular spa types. The relationships between spa types were investigated using the Based Upon Repeat Pattern (BURP) clustering algorithm [42] incorporated into Ridom StaphType. Sequences were analyzed using multiple sequence alignment by the CLUSTALW program (https:/www.genome.jp/tools-bin/clustalw, Kyoto University Bioinformatics Center). 
The primer design for the seven multi-locus sequence typing (MLST) housekeeping genes (arcC, aroE, glpF, gmk, pta, tpi, and yqiL) was obtained from the MLST database (http://www.mlst.net/). We used PCR primers composed of 10 pmol of upstream primer and 10 pmol of downstream primer, $100 \mathrm{ng} / \mathrm{mL}$ of template, and $10 \mathrm{uL}$ of $2 \times \mathrm{iQ}^{\mathrm{TM}} \mathrm{SYBR}^{\circledR}$ Green supermix (Bio-Rad, Hercules, CA, USA). Sterile water was added to achieve a volume of $20 \mathrm{uL}$. PCR cycling was performed as follows: $95^{\circ} \mathrm{C}$ for $5 \mathrm{~min}$; followed by 30 cycles of $94{ }^{\circ} \mathrm{C}$ for $30 \mathrm{~s} ; 55^{\circ} \mathrm{C}$ for $30 \mathrm{~s} ; 72{ }^{\circ} \mathrm{C}$ for $1 \mathrm{~min}$; and a final extension step of $72{ }^{\circ} \mathrm{C}$ for $10 \mathrm{~min}$. The products of seven housekeeping gene fragments were sequenced (Bioneer, Daejeon, Korea) and were compared with allele profiles from the $S$. aureus sample.

MLST database (http://www.mlst.net/) and sequence types (STs) were derived and analyzed with eBURST software (http://saureus.mlst.net/eburst/). Isolates that shared six of seven MLST loci were considered as belonging to the same clonal complex (CC).

Author Contributions: Conceptualization, Y.J.H.; methodology, Y.S.M.; samples performance and validation, Y.S.M.; investigation, Y.S.M.; resources, Y.J.H.; data curation, Y.S.M. and Y.J.H.; writing—original draft preparation, Y.J.H.; writing-review and editing, Y.J.H.; supervision, Y.J.H.; project administration, Y.J.H.; funding acquisition, Y.J.H.

Funding: This study was partially supported by a National Research Foundation grant funded by the Korean government (MSIT, No. 103120) and a Regional Innovation System grant (No. R003942) funded by the Ministry of Trade, Industry, and Energy, Republic of Korea.

Acknowledgments: We thank Yiel-Hea Seo, H.H. Lee, and H.L. Lee who collected the strains and provided technical assistance for the microbiological experiments.

Conflicts of Interest: The authors declare that the research was conducted in the absence of any commercial or financial relationships that could be construed as a potential conflict of interest.

Ethics Statement: The study, in which no human subjects were involved, and only clinical isolates were utilized, meets the exempt category without approval of the Ethics Committee on Human Research of the Health Ministry in South Korea. The study design has not been reviewed by the committee.

\section{References}

1. Diekema, D.J.; Pfaller, M.A.; Shortridge, D.; Zervos, M.; Jones, R.N. Twenty-Year Trends in Antimicrobial Susceptibilities Among Staphylococcus aureus From the SENTRY Antimicrobial Surveillance Program. Open Forum Infect. Dis. 2019, 6, S47-S53. [CrossRef] [PubMed]

2. Chambers, H.F.; DeLeo, F.R. Waves of Resistance: Staphylococcus aureus in the Antibiotic Era. Nat. Rev. Microbiol. 2009, 7, 629. [CrossRef] [PubMed]

3. Tong, S.Y.; Davis, J.S.; Eichenberger, E.; Holland, T.L.; Fowler, V.G. Staphylococcus aureus Infections: Epidemiology, Pathophysiology, Clinical Manifestations, and Management. Clin. Microbiol. Rev. 2015, 28, 603-661. [CrossRef] [PubMed]

4. Peacock, S.J;; Paterson, G.K. Mechanisms of Methicillin-Resistance in Staphylococcus aureus. Ann. Rev. Biochem. 2015, 84, 577-601. [CrossRef] [PubMed]

5. Frenay, H.; Bunschoten, A.; Schouls, L.; Van Leeuwen, W.; Vandenbroucke-Grauls, C.; Verhoef, J.; Mooi, F.R. Molecular Typing of Methicillin-Resistant Staphylococcus aureus on the Basis of Protein A Gene Polymorphism. Eur. J. Clin. Microbiol. Infect. Dis. 1996, 15, 60-64. [CrossRef] [PubMed]

6. Hsu, L.Y.; Harris, S.R.; Chlebowicz, M.A.; Lindsay, J.A.; Koh, T.H.; Krishnan, P.; Tan, T.-Y.; Hon, P.-Y.; Grubb, W.B.; Bentley, S.D.; et al. Evolutionary Dynamics of Methicillin-Resistant Staphylococcus aureus within a Healthcare System. Genome Biol. 2015, 16, 81. [CrossRef] [PubMed]

7. Nübel, U.; Strommenger, B.; Layer, F.; Witte, W. From types to trees: Reconstructing the spatial spread of Staphylococcus aureus based on DNA variation. Int. J. Med. Microbiol. 2011, 301, 614-618. [CrossRef]

8. Harmsen, D.; Claus, H.; Witte, W.; Rothgänger, J.; Claus, H.; Turnwald, D.; Vogel, U. Typing of Methicillin-Resistant Staphylococcus aureus in a University Hospital setting by using Novel Software for spa repeat Determination and Database Management. J. Clin. Microbiol. 2003, 41, 5442-5448. [CrossRef]

9. Goudarzi, M.; Goudarzi, H.; Figueiredo, A.M.S.; Udo, E.E.; Fazeli, M.; Asadzadeh, M.; Seyedjavadi, S.S. Molecular Characterization of Methicillin-Resistant Staphylococcus aureus Strains isolated from Intensive Care Units in Iran: ST22-SCCmec IV/t790 Emerges as the Major Clone. PLoS ONE 2016, 11, e0155529. [CrossRef] 
10. Mazi, W.; Sangal, V.; Sandstrom, G.; Saeed, A.; Yu, J. Evaluation of spa-Typing of Methicillin-Resistant Staphylococcus aureus using High-Resolution Melting Analysis. Inter. J. Infect. Dis. 2015, 38, 125-128. [CrossRef]

11. Murchan, S.; Kaufmann, M.E.; Deplano, A.; de Ryck, R.; Struelens, M.; Zinn, C.E.; Fussing, V.; Salmenlinna, S.; Vuopio-Varkila, J.; El Solh, N.; et al. Harmonization of Pulsed-Field Gel Electrophoresis Protocols for Epidemiological Typing of Strains of Methicillin-Resistant Staphylococcus aureus: A Single Approach Developed by Consensus in 10 European Laboratories and its Application for Tracing the Spread of Related Strains. J. Clin. Microbiol. 2003, 41, 1574-1585. [PubMed]

12. Bosch, T.; de Neeling, A.J.; Schouls, L.M.; Zwaluw, K.W.; Kluytmans, J.A.; Grundmann, H.; Huijsdens, X.W. PFGE Diversity within the Methicillin-Resistant Staphylococcus aureus Clonal Lineage ST. BMC Microbiol. 2010, 10, 40. [CrossRef] [PubMed]

13. Koreen, L.; Ramaswamy, S.V.; Graviss, E.A.; Naidich, S.; Musser, J.M.; Kreiswirth, B.N. spa Typing Method for Discriminating among Staphylococcus aureus Isolates: Implications for Use of a Single Marker to Detect Genetic Micro- and Macrovariation. J. Clin. Microbiol. 2004, 42, 792-799. [CrossRef] [PubMed]

14. Strommenger, B.; Braulke, C.; Heuck, D.; Schmidt, C.; Pasemann, B.; Nübel, U.; Witte, W. spa Typing of Staphylococcus aureus as a Frontline Tool in Epidemiological Typing. J. Clin. Microbiol. 2008, 46, 574-581. [CrossRef] [PubMed]

15. O’Hara, F.P.; Suaya, J.A.; Ray, G.T.; Baxter, R.; Brown, M.L.; Mera, R.M.; Close, N.M.; Thomas, E.; Amrine-Madsen, H. spa Typing and Multilocus Sequence Typing Show Comparable Performance in a Macroepidemiologic Study of Staphylococcus aureus in the United States. Microb. Drug Resist. 2016, 22, 88-96. [CrossRef]

16. Enright, M.C.; Robinson, D.A.; Randle, G.; Feil, E.J.; Grundmann, H.; Spratt, B.G. The evolutionary history of methicillin-resistant Staphylococcus aureus (MRSA). Proc. Natl. Acad. Sci. USA 2002, 99, 7687-7692. [CrossRef]

17. Cho, S.Y.; Chung, D.R. Infection Prevention Strategy in Hospitals in the Era of Community-Associated Methicillin-Resistant Staphylococcus aureus in the Asia-Pacific Region: A Review. Clin. Infect. Dis. 2017, 64, S82-S90. [CrossRef]

18. Asadollahi, P.; Farahani, N.N.; Mirzaii, M.; Khoramrooz, S.S.; van Belkum, A.; Asadollahi, K.; Dadashi, M.; Darban-Sarokhalil, D. Distribution of the Most Prevalent Spa Types among Clinical Isolates of Methicillin-Resistant and -Susceptible Staphylococcus aureus around the World: A Review. Front. Microbiol. 2018, 9, 163. [CrossRef]

19. Sabat, A.J.; Budimir, A.; Nashev, D.; Sá-Leão, R.; van Dijl, J.M.; Laurent, F.; Grundmann, H.; Friedrich, A.W.; ESCMID Study Group of Epidemiological Markers (ESGEM). Overview of Molecular Typing Methods for Outbreak Detection and Epidemiological Surveillance. Eurosurveillance 2013, 18, 20380. [CrossRef]

20. Chen, H.; Liu, Y.; Jiang, X.; Chen, M.; Wang, H. Rapid Change of Methicillin-Resistant Staphylococcus aureus Clones in a Chinese Tertiary Care Hospital over a 15-Year Period. Antimicrob. Agents Chemother. 2010, 54, 1842-1847. [CrossRef]

21. Kim, T.; Yi, J.; Hong, K.H.; Park, J.S.; Kim, E.C. Distribution of Virulence Genes in spa Types of Methicillin-resistant Staphylococcus aureus Isolated from Patients in Intensive Care Units. Korean J. Lab. Med. 2011, 31, 30-36. [CrossRef] [PubMed]

22. Chuang, Y.Y.; Huang, Y.C. Livestock-Associated Meticillin-Resistant Staphylococcus aureus in Asia: An Emerging Issue? Int. J. Antimicrob. Agents 2015, 45, 334-340. [CrossRef] [PubMed]

23. Park, C.; Lee, D.G.; Choi, S.M.; Park, S.H.; Choi, J.H.; Yoo, J.H.; Hur, J.A.; Shin, W.S. A Case of Perianal Abscess due to Panton-Valentine Leukocidin Positive Community-Associated Methicillin-Resistant Staphylococcus aureus: Report in Korea and Literature Review from the Far East. Infect. Chemother. 2008, 40, 121-126. [CrossRef]

24. Ahmad, N.; Ruzan, I.N.; Abd, G.M.K.; Hussin, A.; Nawi, S.; Aziz, M.N.; Maning, N.; Eow, V.L.K. Characteristics of Community- and Hospital-acquired Meticillin-Resistant Staphylococcus aureus Strains carrying SCCmec type IV isolated in Malaysia. J. Med. Microbiol. 2009, 58, 1213-1218. [CrossRef] [PubMed]

25. Song, J.H.; Hsueh, P.R.; Chung, D.R.; Ko, K.S.; Kang, C.I.; Peck, K.R.; Yeom, J.S.; Kim, S.W.; Chang, H.H.; Kim, Y.S.; et al. Spread of Methicillin-Resistant Staphylococcus aureus between the Community and the Hospitals in Asian Countries: An ANSORP Study. J. Antimicrob. Chemother. 2011, 66, 1061-1069. [CrossRef] [PubMed] 
26. Chen, Y.; Liu, Z.; Duo, L.; Xiong, J.; Gong, Y.; Yang, J.; Wang, Z.; Wu, X.; Lu, Z.; Meng, X.; et al. Characterization of Staphylococcus aureus from Distinct Geographic Locations in China: An Increasing Prevalence of spa-t030 and SCCmec Type III. PLoS ONE 2014, 9, e96255. [CrossRef]

27. Japoni-Nejad, A.; Rezazadeh, M.; Kazemian, H.; Fardmousavi, N.; van Belkum, A.; Ghaznavi-Rad, E. Molecular Characterization of the First Community-Acquired Methicillin-Resistant Staphylococcus aureus Strains from Central Iran. Int. J. Infect. Dis. 2013, 17, e949-e954. [CrossRef]

28. Cha, H.Y.; Moon, D.C.; Choi, C.H.; Oh, J.Y.; Jeong, Y.S.; Lee, Y.C.; Seol, S.Y.; Cho, D.T.; Chang, H.-H.; Kim, S.-W.; et al. Prevalence of the ST239 clone of methicillin-resistant Staphylococcus aureus and differences in antimicrobial susceptibilities of ST239 and ST5 clones identified in a Korean hospital. J. Clin. Microbiol. 2005, 43, 3610-3614. [CrossRef]

29. Chong, Y.P.; Kim, E.S.; Park, S.J.; Park, K.H.; Kim, T.; Kim, M.N.; Kim, S.H.; Lee, S.O.; Choi, S.H.; Woo, J.H.; et al. Accessory gene regulator (agr) dysfunction in Staphylococcus aureus bloodstream isolates from South Korean patients. Antimicrob. Agents Chemother. 2013, 57, 1509-1512. [CrossRef]

30. Li, J.; Wang, L.; Ip, M.; Sun, M.; Sun, J.; Huang, G.; Wang, C.; Deng, L.; Zheng, Y.; Fu, Z.; et al. Molecular and clinical characteristics of clonal complex 59 methicillin-resistant Staphylococcus aureus infections in Mainland China. PLOS ONE 2013, 8, e70602. [CrossRef]

31. Chen, C.J.; Huang, Y.C. New epidemiology of Staphylococcus aureus infecion in Asia. Clin. Microbiol. Infect. 2014, 20, 605-623. [CrossRef] [PubMed]

32. Geng, W.; Yang, Y.; Wu, D.; Huang, G.; Wang, C.; Deng, L.; Zheng, Y.; Fu, Z.; Li, C.; Shang, Y.; et al. Molecular characteristics of community-acquired, methicillin-resistant Staphylococcus aureus isolated from Chinese children. FEMS Immunol. Med. Microbiol. 2010, 58, 356-362. [CrossRef] [PubMed]

33. DeLeo, F.R.; Otto, M.; Kreiswirth, B.N.; Chambers, H.F. Community-associated meticillin-resistant Staphylococcus aureus. Lancet 2010, 375, 1557-1568. [CrossRef]

34. Chung, G.; Cha, J.; Han, S.; Jang, H.; Lee, K.; Yoo, J.; Yoo, J.; Kim, H.; Eun, S.; Kim, B.; et al. Nationwide surveillance study of vancomycin intermediate Staphylococcus aureus strains in Korean hospitals from 2001 to 2006. J. Microbiol. Biotechnol. 2010, 20, 637-642. [PubMed]

35. Park, J.Y.; Jin, J.S.; Kang, H.Y.; Jeong, E.H.; Lee, J.C.; Lee, Y.C.; Seol, S.Y.; Cho, D.T.; Kim, J. A comparison of adult and pediatric methicillin-resistant Staphylococcus aureus isolates collected from patients at a university hospital in Korea. J. Microbiol. 2007, 45, 447-452.

36. Sung, J.Y.; Lee, J.; Choi, E.H.; Lee, H.J. Changes in molecular epidemiology of community-associated and health care-associated methicillin-resistant Staphylococcus aureus in Korean children. Diagn. Microbiol. Infect. Dis. 2012, 74, 28-33. [CrossRef]

37. Joo, E.J.; Choi, J.Y.; Chung, D.R.; Song, J.H.; Ko, K.S. Characteristics of the community-genotype sequence type 72 methicillin-resistant Staphylococcus aureus isolates that underlie their persistence in hospitals. J. Microbiol. 2016, 54, 445-450. [CrossRef]

38. Wayne, P.A. Clinical and laboratory standards institute. Performance standards for antimicrobial susceptibility testing, 23th ed.; 2013.

39. Varaldo, P.E.; Montanari, M.P.; Giovanetti, E. Genetic Elements Responsible for Erythromycin Resistance in Streptococci. Antimicrob. Agents Chemother. 2009, 53, 343-353. [CrossRef]

40. Zhang, K.; McClure, J.A.; Elsayed, S.; Louie, T.; Conly, J.M. Novel Multiplex PCR Assay for Characterization and Concomitant Subtyping of Staphylococcal Cassette Chromosome mec Types I to V in Methicillin-Resistant Staphylococcus aureus. J. Clin. Microbiol. 2005, 43, 5026-5033. [CrossRef]

41. Strommenger, B.; Kettlitz, C.; Werner, G.; Witte, W. Multiplex PCR Assay for Simultaneous Detection of Nine Clinically Relevant Antibiotic Resistance Genes in Staphylococcus aureus. J. Clin. Microbiol. 2003, 41, 4089-4094. [CrossRef]

42. Mellmann, A.; Weniger, T.; Berssenbrügge, C.; Rothgänger, J.; Sammeth, M.; Stoye, J.; Harmsen, D. Based Upon Repeat Pattern (BURP): An Algorithm to Characterize the Long-Term Evolution of Staphylococcus aureus Populations based on spa Polymorphisms. BMC Microbiol. 2007, 7, 98. [CrossRef] [PubMed]

(C) 2019 by the authors. Licensee MDPI, Basel, Switzerland. This article is an open access article distributed under the terms and conditions of the Creative Commons Attribution (CC BY) license (http://creativecommons.org/licenses/by/4.0/). 